\title{
Generalized gradient flow equation and its application to super Yang-Mills theory
}

\author{
Kengo Kikuchi ${ }^{a}$ and Tetsuya Onogi ${ }^{b}$ \\ ${ }^{a}$ Yukawa Institute for Theoretical Physics, Kyoto University, \\ Kyoto, 606-8502 Japan \\ ${ }^{b}$ Department of Physics, Osaka University, \\ Toyonaka, 560-0043 Japan \\ E-mail: kengo@yukawa.kyoto-u.ac.jp, onogi@phys.sci.osaka-u.ac.jp
}

ABSTRACT: We generalize the gradient flow equation for field theories with nonlinearly realized symmetry. Applying the formalism to super Yang-Mills theory, we construct a supersymmetric extension of the gradient flow equation. It can be shown that the super gauge symmetry is preserved in the gradient flow. Furthermore, choosing an appropriate modification term to damp the gauge degrees of freedom, we obtain a gradient flow equation which is closed within the Wess-Zumino gauge.

KeYwords: Lattice Gauge Field Theories, Lattice Quantum Field Theory, Supersymmetric gauge theory

ArXiv EPRINT: 1408.2185 


\section{Contents}

1 Introduction $\quad 2$

2 Gradient flow equation for Yang-Mills theory 3

3 General form of gradient flow equation 4

3.1 Field theories with nonlinearly realized symmetry 4

3.2 Our proposal for generalized gradient flow equation 5

$\begin{array}{lll}4 & \text { Supersymmetric gradient flow equation } & 6\end{array}$

4.1 Derivation of gradient flow equation of super Yang-Mills theory 6

4.2 Symmetries of gradient flow equation 8

5 Gradient flow equation of super Yang-Mills theory under Wess-Zumino gauge

6 Gradient flow equation of super Yang-Mills theory for each component 9

$\begin{array}{lll}\text { 6.1 Expansion in component fields } & 10\end{array}$

6.2 Gradient flow equation of super Yang-Mills theory for each component of vector multiplet

7 Summary and discussion $\quad 12$

$\begin{array}{ll}\text { A Notation } & 13\end{array}$

$\begin{array}{ll}\text { B } O(N) \text { nonlinear sigma model } & 14\end{array}$

$\begin{array}{ll}\text { C Lattice gauge theory } & 15\end{array}$

$\begin{array}{ll}\text { D Short summary of supersymmetry } & 17\end{array}$

$\begin{array}{ll}\text { D.1 Definition } & 17\end{array}$

$\begin{array}{lll}\text { D.2 Wess-Zumino gauge } & 18\end{array}$

$\begin{array}{lll}\text { E Derivation of gradient flow equation for vector superfield } \mathcal{V} & 18\end{array}$

$\begin{array}{ll}\text { F Pure abelian supersymmetric theory } & \mathbf{2 0}\end{array}$

F.1 Derivation of gradient flow equation of pure abelian supersymmetric theory 20

F.2 Gradient flow equation of pure Yang-Mills theory for each component of vector multiplet 21

F.3 Flow time dependence of super gauge transformation 22

G Expansion of equation (6.1) with component fields $\quad 22$

G.1 Coordinate transformation $\quad 22$

$\begin{array}{lll}\text { G.2 Useful formulae } & 23\end{array}$ 


\section{Introduction}

In recent years, the gradient flow equation has been the focus of much attention. The equation was proposed by Martin Luscher [1] for Yang-Mills theory as a certain type of diffusion equation to give a one parameter deformation of the gauge field evolving in the flow time starting from the bare gauge field as the initial condition. It was found [2] that the expectation value of any gauge invariant local operators of the new gauge field, which is the solution of the gradient flow equation, is finite without additional renormalization.

Various applications of the physical observable are studied recently. Ref. [3] give a review of the recent applications. For example, the gradient flow of a matter field $\chi$ is proposed as follows [4]:

$$
\begin{aligned}
\dot{\chi} & =\Delta \chi,\left.\quad \chi\right|_{t=0}=\psi, \\
\Delta & =\not D^{2} \quad \text { or simply } \quad \Delta=D_{\mu} D_{\mu},
\end{aligned}
$$

where $D_{\mu}=\partial_{\mu}+B_{\mu}$. Using this equation, the expectation value of the chiral densities is calculated [4]. The relation between the small flow time behavior of certain gauge invariant local products and the correctly-normalized conserved energy-momentum tensor in the Yang-Mills theory is given [5]. More appropriate probes for the translation Ward identities is defined [6]. The methods is also applied in the lattice theory [1,7-21], a new scheme of the step scaling, the improved action, and so on.

In this way, the gradient flow equation has spurred a great deal of research. In view of this nice property, it is natural to consider possible extensions of this method for other theories. One interesting system is the super Yang-Mills theory. This theory shares similar property as the Quantum Chromodynamics (QCD) since it has a matter field called gaugino, though it is in the adjoint representation. On the other hand, the restriction from the supersymmetry (SUSY) can give a natural extension of the gradient flow in Yang-Mills theory. In this sense, super Yang-Mills theory could be an interesting laboratory for studying the extension of the gradient flow equation.

Once it is constructed, there can be interesting applications of the supersymmetric Yang-Mills lattice theory. For example, imposing the supersymmetric Ward-Takahashi identity for composite fields with finite flow time, one might be able to determine the renormalization factor for the super current as well as various improvement terms in the action and operators, in close analogy with Luscher's work on the chiral symmetry in QCD with Wilson fermions. This method may also be useful for testing the validity of various proposals of supersymmetric lattice models.

In this paper, we construct the gradient flow equation for super Yang-Mills theory. Since the super gauge symmetry is nonlinearly realized, we first construct the generalization of the gradient flow equation for quantum field theories with nonlinearly realized symmetry. Applying the generalized equation to super Yang-Mills theory, we construct a natural extension of the gradient flow using superfield formalism. We find that with a special choice of the modification term in the gradient flow equation, we obtain a closed equation within the Wess-Zumino (WZ) gauge. 
This paper is organized as follows. In section 2, we give a brief review of the method of gradient flow in Yang-Mills theory. In section 3 we propose the generalized gradient flow equation of the quantum field theory with nonlinearly realized symmetry. In section 4 we apply the generalized equation to super Yang-Mills theory. To obtain the compact expression of the equation of super Yang-Mills theory, we look for a special choice of the modification term to give a closed equation within the WZ gauge. In section 6 , we give the gradient flow equation for super Yang-Mills theory concretely with component fields including the gaugino field. Section 7 is devoted to summary and discussions.

\section{Gradient flow equation for Yang-Mills theory}

The gauge field $B_{\mu}$ is defined by the gradient flow equation

$$
\begin{aligned}
& \dot{B}_{\mu}=D_{\nu} G_{\nu \mu}+\alpha_{0} D_{\mu} \partial_{\nu} B_{\nu}, \\
& \left.B_{\mu}\right|_{t=0}=A_{\mu},
\end{aligned}
$$

where the dot means a differential in terms of the flow time $t, A_{\mu}$ describe a fundamental bare field of $\mathrm{SU}(N)$ gauge theory, $G_{\mu \nu}$ and $D_{\mu}$ are defined by

$$
\begin{aligned}
G_{\mu \nu} & =\partial_{\mu} B_{\nu}-\partial_{\nu} B_{\mu}+\left[B_{\mu}, B_{\nu}\right], \\
D_{\mu} & =\partial_{\mu}+\left[B_{\mu}, \cdot\right]
\end{aligned}
$$

respectively. The reason why we call the equation the gradient flow one is that the first term of r.h.s. of eq. (2.1) is proportional to the gradient of the action,

$$
S=\int d^{4} x \operatorname{Tr}\left[G_{\mu \nu}(x) G_{\mu \nu}(x)\right] .
$$

The second term of the r.h.s. of eq. (2.1) is a modification term to damp the gauge degrees of freedom. In this paper, we call this term as the $\alpha_{0}$ term. It has to be proportional to the form of gauge transformation so that it does not affect the evolution of gauge invariant observables. Luscher claims that any expectation value which is described by the gauge field $B_{\mu}$, which is defined by eq. (2.1) at positive flow time has a well-defined continuum limit without additional renormalization. Calculating the expectation value of the energy density at one loop order using this method, he showed that it is the case [1]. Soon after that, Luscher and Weisz proved this claim to all order in perturbation theory [2]. Hereafter, we call the claim of the all order finiteness of the observables constructed from the gauge field $B_{\mu}$ with finite flow time as "the Luscher-Weisz theorem". Eq. (2.1) has a gauge symmetry at any flow time if the gauge parameter $\omega(t, x)$ satisfies the condition

$$
\partial_{t} \omega=\alpha_{0} D_{\mu} \partial_{\mu} \omega
$$

The symmetry of the equation is a key to prove the Luscher-Weisz theorem.

When we extend the method of gradient flow to the super Yang-Mills theory, we encounter a problem. Since the super gauge symmetry is nonlinearly realized, the naive gradient flow equation does not respect the super gauge symmetry. In order to solve this problem, we propose a generalization of the gradient flow equation with nonlinearly realized symmetry in the next section. 


\section{General form of gradient flow equation}

The gradient flow gives the steepest descent time evolution in the space based on the energy function of the system. For example, when the space is $\mathbf{R}^{n}$, the gradient flow equation is given as

$$
\frac{d q^{i}}{d t}=-\frac{\partial E(q)}{\partial q^{i}} \quad(i=1, \cdots, n)
$$

where $q^{i}(i=1, \cdots, n)$ is the position in $\mathbf{R}^{n}$ and $E(q)$ is the energy function. The time evolution of the energy function is then given as

$$
\frac{d E(q)}{d t}=\sum_{i=1}^{n} \frac{\partial E(q)}{\partial q^{i}} \frac{d q^{i}}{d t}=-\sum_{i=1}^{n}\left(\frac{\partial E(q)}{\partial q^{i}}\right)^{2} \leq 0,
$$

so that the energy decreases monotonically in time towards an extremum of the energy function.

The gradient flow can be naturally extended to field theory. For example, in the field theory in 4 dimension with field $\phi(x)$ with action $S(\phi)$, taking the space $\{\phi(x)\}$ as the functional space and the action $S(\phi)$ as the energy function the gradient flow equation becomes

$$
\frac{\partial \phi_{t}(x)}{\partial t}=-\frac{\delta S\left(\phi_{t}\right)}{\delta \phi_{t}(x)}
$$

Since the flow stops when the field reaches the extremum of the action, the gradient flow gives an interpolation of the initial field and a classical solution of the theory.

There is a question whether the symmetry of the theory is preserved under the time evolution with the gradient flow equation of the type in eq. (3.3). Fortunately, in YangMills theory case, the gauge symmetry is preserved. However, in general, the problem can arise when the symmetry of the system is nonlinearly realized.

It turns out that the super Yang-Mills theory has a super gauge symmetry which is nonlinearly realized with respect to the vector superfield. Since the BRS symmetry is the key for Luscher-Weisz theorem for Yang-Mills theory, one can expect that the super gauge symmetry in super Yang-Mills theory could also play a crucial role. In the following subsections, we consider the field theories with a nonlinearly realized symmetry and construct the generalization of the gradient flow equation which respects the symmetry.

\subsection{Field theories with nonlinearly realized symmetry}

Let us now generalize the gradient flow for field theories with a symmetry which is nonlinearly realized.

$$
\phi^{a}(x) \rightarrow \phi^{\prime a}(x), \quad(a=1, \cdots, M)
$$

where $a$ is the index for the internal degrees of freedom and $M$ is the total number of components. $\phi^{\prime}$ is a nonlinear function of $\phi$. Under this transformation, the action is invariant

$$
S\left(\phi^{\prime}\right)=S(\phi)
$$


One can find that the naive gradient flow equation given in eq. (3.3) does not keep the symmetry. This is because the gradient flow equation based on eq. (3.3) for the field after the transformation $\phi^{\prime}$ reads

$$
\frac{\partial \phi_{t}^{a \prime}(x)}{\partial t}=-\frac{\delta S\left(\phi_{t}^{\prime}\right)}{\delta \phi_{t}^{a \prime}(x)}
$$

Using eq. (3.5) and the chain rule for derivative, the above equation becomes

$$
\sum_{b=1}^{M} \frac{\partial \phi_{t}^{a \prime}(x)}{\partial \phi_{t}^{b}(x)} \frac{\partial \phi_{t}^{b}(x)}{\partial t}=-\sum_{b=1}^{M} \frac{\partial \phi_{t}^{b}(x)}{\partial \phi_{t}^{a \prime}(x)} \frac{\delta S\left(\phi_{t}\right)}{\delta \phi_{t}^{b}(x)} .
$$

Multiplying $\frac{\partial \phi_{t}^{c}(x)}{\partial \phi_{t}^{\prime \prime}(x)}$ and sum over $a$, the naive gradient flow equation for $\phi_{t}^{\prime}$ would reduce to

$$
\frac{\partial \phi_{t}^{c}(x)}{\partial t}=-\sum_{a=1}^{M} \frac{\partial \phi_{t}^{c}(x)}{\partial \phi_{t}^{a \prime}(x)} \frac{\partial \phi_{t}^{b}(x)}{\partial \phi_{t}^{a \prime}(x)} \frac{\delta S\left(\phi_{t}\right)}{\delta \phi_{t}^{b}(x)} .
$$

Thus the naive gradient flow equations before and after the symmetry transformation are different.

\subsection{Our proposal for generalized gradient flow equation}

How can we define a gradient flow equation which respects the symmetry? As we have seen in the previous subsection, the problem in the naive gradient flow equation is that the l.h.s. and the r.h.s. transform differently under the symmetry transformation. A natural solution could be to introduce a "metric" to compensate the mismatch of the transformation property. The metric in functional space for the field theory in $D$ dimension can be defined through the norm of the variation of fields $\delta \phi(x)$ which is invariant under the symmetry.

$$
\|\delta \phi\|^{2}=\int d^{D} x g_{a b}(\phi(x)) \delta \phi^{a}(x) \delta \phi^{b}(x), \quad a=1,2, \cdots, M
$$

where $M$ is the number of components of the field and $g_{a b}(\phi(x))$ is the metric in the functional space. The metric should be chosen in such a way that the norm is invariant under the symmetry transformation as

$$
\left\|\delta \phi^{\prime}\right\|^{2}=\|\delta \phi\|^{2}
$$

which leads to the following properties for the metric in the functional space.

$$
\begin{aligned}
& g_{a b}\left(\phi^{\prime}(x)\right)=\frac{\partial \phi^{c}(x)}{\partial \phi^{\prime a}(x)} \frac{\partial \phi^{d}(x)}{\partial \phi^{\prime b}(x)} g_{c d}(\phi(x)), \\
& g^{a b}\left(\phi^{\prime}(x)\right)=\frac{\partial \phi^{\prime a}(x)}{\partial \phi^{c}(x)} \frac{\partial \phi^{\prime b}(x)}{\partial \phi^{d}(x)} g^{c d}(\phi(x)) .
\end{aligned}
$$

Whether one can find an appropriate metric or not for a given field theory is quite nontrivial, but there are quite a few examples in which one can find the metric explicitly such as $O(N)$ nonlinear sigma model or $\mathrm{SU}(N)$ lattice gauge theory. In any case, eqs. (3.11) and (3.12) 
mean that symmetry transformation is the isometry for the metric defined through the invariant norm.

The condition for the isometry in eq. (3.12) gives exactly the right quantity to compensate the mismatch of the transformation property in the naive gradient flow equation. Thus we find that when we require the invariance of the gradient flow under the symmetry, the gradient flow should be modified as

$$
\frac{\partial \phi_{t}^{a}(x)}{\partial t}=-g^{a b}\left(\phi_{t}(x)\right) \frac{\delta S\left(\phi_{t}\right)}{\delta \phi_{t}^{b}(x)} .
$$

In what follows, we call the above equation as the generalized gradient flow equation. It is clear the time evolutions of $\phi$ and $\phi^{\prime}$ fields with our generalized gradient flow equation are mutually consistent under symmetry transformation The evolution equation (3.13) was also discussed in the context of Fokker-Plank equation. [23-30].

In appendices $\mathrm{B}$ and $\mathrm{C}$, we apply the generalized gradient flow equation to the $O(N)$ nonlinear sigma model and the $\mathrm{SU}(N)$ lattice gauge theory and verify its validity. We find that the generalized gradient flow equation gives a time evolution which respects the nonlinearly realized symmetry of the system. In the next section, we construct the gradient flow equation of the super Yang-Mills theory based on the generalized gradient flow equation which respects the super gauge symmetry.

\section{Supersymmetric gradient flow equation}

\subsection{Derivation of gradient flow equation of super Yang-Mills theory}

Before studying the super Yang-Mills theory, let us review the steps for constructing the gradient flow equation in ordinary non-SUSY Yang-Mills theory. The local gauge transformation is given as

$$
A_{\mu}(x) \rightarrow A_{\mu}(x)+D_{\mu} \omega(x),
$$

where $D_{\mu}$ is the covariant derivative and $\omega(x)$ is the gauge transformation parameter. One can see that the invariant norm of the vector field $\delta A_{\mu}(x)$ is given as

$$
\left\|\delta A_{\mu}(x)\right\|^{2}=\int d^{4} x \operatorname{Tr}\left[\delta A_{\mu}(x) \delta A_{\mu}(x)\right],
$$

which means that the metric in the field space is

$$
g^{a b}\left(A_{\mu}\right)=2 \delta^{a b} .
$$

Therefore, how to derive the gradient flow in ordinary Yang-Mills theory can be summarized as follows:

1. Starting from the Yang-Mills action $S_{\mathrm{YM}}$, we make a variation over the $A_{\mu}^{b}(x)$ field, and multiply the metric $2 \delta^{a b}$, where

$$
\begin{aligned}
S_{\mathrm{YM}} & =\int d^{4} x \operatorname{Tr}\left[F_{\mu \nu}(x) F_{\mu \nu}(x)\right], \\
F_{\mu \nu} & =\partial_{\mu} A_{\nu}-\partial_{\nu} A_{\mu}+\left[A_{\mu}, A_{\nu}\right] .
\end{aligned}
$$


2. We replace the $A_{\mu}(x)$ field with the new gauge field $B_{\mu}(t, x)$, and impose the initial condition $B_{\mu}(0, x)=A_{\mu}(x)$, and introduce the field strength $G_{\mu \nu} \equiv \partial_{\mu} B_{\nu}-\partial_{\nu} B_{\mu}+$ $\left[B_{\mu}, B_{\nu}\right]$.

3. We add a new gauge fixing term to suppress the increase of the degree of new gauge freedom in the flow time direction. It has to be proportional to the gauge transformation, because physical quantities do not depend on the term.

4. We regard the sum of them as r.h.s. of the gradient flow equation.

5. We regard the derivative of $B_{\mu}^{a}(t, x)$ with respect to $t$ as l.h.s. of the gradient flow equation.

Thus, we obtain the gradient flow equation in Yang-Mills theory as eqs. (2.1) and (2.2).

We now apply the general gradient flow equation to super Yang-Mills theory. The super gauge transformation of the super Yang-Mills vector superfield $V$ is given as

$$
e^{V} \rightarrow e^{-i \Lambda^{\dagger}} e^{V} e^{i \Lambda}
$$

where $\Lambda, \Lambda^{\dagger}$ are arbitrary chiral and anti-chiral superfields. The component of superfield $V$ is defined by $V=\left\{C, X, \bar{X}, M, M^{*}, V_{m}, \Lambda, \bar{\Lambda}, D\right\}$. The invariant norm for $\delta V$ under the super gauge transformation is then given as

$$
\|\delta V\|^{2} \equiv-\int d^{8} z \operatorname{Tr}\left[e^{-V}\left(\delta e^{V}\right) e^{-V}\left(\delta e^{V}\right)\right] .
$$

This means that the space of vector superfields has a nontrivial metric in functional space. To obtain the gradient flow equation of the super Yang-Mills theory, we replace the statement partly as follows:

- Yang-Mills action $S_{\mathrm{YM}} \rightarrow$ Super Yang-Mills action $S_{\mathrm{SYM}}$, where

$$
\begin{aligned}
S_{\mathrm{SYM}} & =-\int d^{4} x \int d^{2} \theta \operatorname{Tr}\left[W^{\alpha} W_{\alpha}\right]+\text { h.c. }, \\
W_{\alpha} & =-\bar{D} \bar{D}\left(e^{-V} D_{\alpha} e^{V}\right) .
\end{aligned}
$$

- Gauge field $A_{\mu}(x) \rightarrow$ Superfield $V(z)$. The argument $z$ stands for super coordinate $(x, \theta, \bar{\theta})$.

- New gauge field $B_{\mu}(t, x) \rightarrow$ New superfield $\mathcal{V}(t, z)$. The component of superfield $\mathcal{V}$ is defined by $\mathcal{V}=\left\{c, \chi, \bar{\chi}, m, m^{*}, v_{m}, \lambda, \bar{\lambda}, d\right\}$. We impose the initial condition $\mathcal{V}(0, z)=V(z)$.

- Gauge transformation $\rightarrow$ Super gauge transformation.

- Metric $g^{a b}\left(A_{\mu}\right) \rightarrow g^{a b}(V)$. 
Thus we propose a general form of the supersymmetric extension of the gradient flow equation,

$$
\frac{\partial \mathcal{V}^{a}}{\partial t}=-g^{a b}(\mathcal{V}) \frac{\delta S_{\mathrm{SYM}}}{\delta \mathcal{V}^{b}}+\alpha_{0} \delta \mathcal{V}^{a}
$$

The $\delta \mathcal{V}$ is the super gauge transformation of $\mathcal{V}$, which is defined by the equation as

$$
\delta \mathcal{V}=L_{\mathcal{V} / 2} \cdot\left[\left(\Phi-\Phi^{\dagger}\right)+\operatorname{coth}\left(L_{\mathcal{V} / 2}\right) \cdot\left(\Phi+\Phi^{\dagger}\right)\right]
$$

where $\Phi$ is a chiral superfield. Substituting the explicit forms of $g^{a b}(\mathcal{V}), \frac{\delta S_{S Y M}}{\delta \mathcal{V}^{b}}, \delta \mathcal{V}_{a}$, we obtain the gradient flow equation in the matrix form as

$$
\frac{\partial \mathcal{V}}{\partial t}=\frac{L_{\mathcal{V}}}{1-e^{-L_{\mathcal{V}}}}\left(F+\alpha_{0} \Phi_{\mathcal{V}}\right)+\text { h.c. }
$$

where

$$
F=D^{\alpha} w_{\alpha}+\left\{e^{-\mathcal{V}} D^{\alpha} e^{\mathcal{V}}, w_{\alpha}\right\}
$$

and $\Phi_{\mathcal{V}}$ is a chiral field, $\mathcal{V}=\mathcal{V}^{a} T^{a}$ and $T^{a}$ is a representation matrix. The field strength $w_{\alpha}$ is given by $w_{\alpha} \equiv-\bar{D} \bar{D}\left(e^{-\mathcal{V}} D_{\alpha} e^{\mathcal{V}}\right)$. The $L_{\mathcal{V}}$ is defined by

$$
L_{\mathcal{V}} \cdot \equiv[\mathcal{V}, \cdot]
$$

The derivation of the above equation is given in appendix E. The covariant term of eq. (4.12) was also discussed in the stochastic quantization [31, 32]. We can also rewrite eq. (4.12) more simply using $e^{\mathcal{V}}$ as a basic variable as

$$
\frac{\partial e^{\mathcal{V}}}{\partial t}=e^{\mathcal{V}}\left(F+\alpha_{0} \Phi_{\mathcal{V}}\right)+h . c . .
$$

This form is useful for studying the time dependence of the super gauge transformation as discussed in the next subsection.

\subsection{Symmetries of gradient flow equation}

We comment on the supersymmetry and super gauge symmetry of the gradient flow equation. The equation consists of covariant derivative operators $D, \bar{D}$, and vector multiplet $\mathcal{V}$. Because supersymmetric transformation operators $Q \xi, \bar{Q} \bar{\xi}$ commute with $D, \bar{D}$, the equation keeps SUSY manifestly if $\xi$ and $\bar{\xi}$ do not depend on the flow time.

It is important to examine the condition that the gradient flow equation has super gauge symmetry at any flow time. Taking the infinitesimal super gauge transformation for both sides of the gradient flow equation, we obtain the condition for $\Lambda$,

$$
i \frac{d \Lambda}{d t}=\alpha_{0}\left(\delta_{\Lambda} \Phi_{\mathcal{V}}+i\left[\Lambda, \Phi_{\mathcal{V}}\right]\right)
$$

The $\delta_{\Lambda}$ is infinitesimal super gauge transformation,

$$
\Phi_{\mathcal{V}} \rightarrow \Phi_{\mathcal{V}}+\delta_{\Lambda} \Phi_{\mathcal{V}}
$$

If $\Lambda$ satisfies the condition eq. (4.16), the gradient flow equation is invariant for the super gauge transformation at any flow time. 


\section{Gradient flow equation of super Yang-Mills theory under Wess-Zumino gauge}

In this section, we determine the form of the gradient flow equation of super Yang-Mills theory under the WZ gauge. Because eq. (4.12) have infinite number of terms, it is very difficult to solve it. In order to obtain the flow equation with finite number of terms, we choose the WZ gauge.

However, generally the time evolution from the flow equation can carry the system away from the WZ gauge. Therefore, the most important question is whether there exists the special chiral field $\Phi_{\mathcal{V}}$ which give the super gauge transformation keeping the WZ gauge. As a result, we find that such a $\Phi_{\mathcal{V}}$ exists.

Here we discuss how to determine the form of the $\alpha_{0}$ term. We try to find out the special form of the $\alpha_{0}$ term so that the gradient flow equation is consistent within the WZ gauge. This means the $\alpha_{0}$ term has to satisfy the following requirements.

- It is positive.

- The mass dimension is two.

- It is described by super gauge transformation $\delta \mathcal{V}$.

- The flow of the vector field keeps the WZ gauge at any flow time.

As a result, we found out that there exists at least one example of the $\alpha_{0}$ term which satisfies these conditions.

$$
\begin{aligned}
\alpha_{0} & =1 \\
\delta \mathcal{V} & =\Phi_{\mathcal{V}}+\Phi_{\mathcal{V}}^{\dagger}+\frac{1}{2}\left[\mathcal{V}, \Phi_{\mathcal{V}}-\Phi_{\mathcal{V}}^{\dagger}\right]+\frac{1}{12}\left[\mathcal{V},\left[\mathcal{V}, \Phi_{\mathcal{V}}+\Phi_{\mathcal{V}}^{\dagger}\right]\right]
\end{aligned}
$$

where

$$
\Phi_{\mathcal{V}}=\bar{D}^{2}\left(D^{2} \mathcal{V}+\left[D^{2} \mathcal{V}, \mathcal{V}\right]\right)
$$

It is possible that $\Phi_{\mathcal{V}}$ which gives the super gauge transformation keeping the WZ gauge may not be unique. However, this example can be useful for further studies.

\section{Gradient flow equation of super Yang-Mills theory for each component}

In this section, we applied our equation, which is obtained in section 4, to super YangMills theory concretely, and derive the gradient flow equation of each component under WZ gauge. It gives the gradient flow equation of the matter field. For the sake of understanding this section, we give the equation in the case of the pure Abelian supersymmetric theory in appendix $\mathrm{F}$ as an example. 


\subsection{Expansion in component fields}

We rewrite $F$ for the convenience as

$$
F=D^{\alpha} w_{\alpha}+\left\{e^{-\mathcal{V}} D^{\alpha} e^{\mathcal{V}}, w_{\alpha}\right\} .
$$

Useful formulae to expand eq. (6.1) in component fields are given in appendix G. The gauge covariant term is given as

$$
\begin{aligned}
& \left(\frac{L_{\mathcal{V}}}{1-e^{-L_{\mathcal{V}}}} \cdot F\right)+\left(\frac{L_{\mathcal{V}}}{e^{L_{\mathcal{V}}}-1} \cdot F^{\dagger}\right) \\
& \quad=F+F^{\dagger}+\frac{1}{2}\left[\mathcal{V}, F-F^{\dagger}\right]+\frac{1}{12}\left[\mathcal{V},\left[\mathcal{V}, F+F^{\dagger}\right]\right]+O\left(\mathcal{V}^{3}\right)
\end{aligned}
$$

where $F$ is represented in $(x, \theta, \bar{\theta})$ coordinates by

$$
\begin{aligned}
F(x, \theta, \bar{\theta})= & -8 d+8 \theta \sigma^{m} \mathscr{D}_{m} \bar{\lambda}-8 \bar{\theta} \bar{\sigma}^{m} \mathscr{D}_{m} \lambda \\
& +4\left(\bar{\theta} \bar{\sigma}^{m} \theta\right)\left[v_{m}, d\right]+4\left(\theta \sigma^{k} \bar{\sigma}^{m} \sigma^{l} \bar{\theta}\right) \mathscr{D}_{l} v_{m k}+8[\bar{\theta} \bar{\lambda}, \theta \lambda] \\
& -8 i \theta \theta\left(\bar{\theta} \bar{\sigma}^{l} \sigma^{m} \mathscr{D}_{l} \mathscr{D}_{m} \bar{\lambda}\right)+8 i \theta \theta[\bar{\theta} \bar{\lambda}, d] \\
& +4 i \theta \theta\left(\bar{\theta} \bar{\sigma}^{k} \sigma^{m} \partial_{k} \mathscr{D}_{m} \bar{\lambda}\right)+4 i \bar{\theta} \bar{\theta}\left(\theta \sigma^{k} \bar{\sigma}^{m} \partial_{k} \mathscr{D}_{m} \lambda\right) \\
& +\theta \theta \bar{\theta} \bar{\theta}\left(2 \square d+2 i \partial^{m}\left[v_{m}, d\right]+i \operatorname{Tr}\left[\bar{\sigma}^{m} \sigma^{l} \bar{\sigma}^{n} \sigma^{k}\right] \partial_{n} \mathscr{D}_{l} v_{m k}\right. \\
& \left.-2 i \partial_{m}\left\{\bar{\lambda}_{\dot{\alpha}},\left(\bar{\sigma}^{m} \lambda\right)^{\dot{\alpha}}\right\}\right) .
\end{aligned}
$$

On the other hand, $F^{\dagger}$ is represented in $(x, \theta, \bar{\theta})$ coordinates by

$$
\begin{aligned}
F^{\dagger}(x, \theta, \bar{\theta})= & -8 d+8 \theta \sigma^{m} \mathscr{D}_{m} \bar{\lambda}-8 \bar{\theta} \bar{\sigma}^{m} \mathscr{D}_{m} \lambda \\
& -4\left(\bar{\theta} \bar{\sigma}{ }^{m} \theta\right)\left[v_{m}, d\right]+4\left(\theta \sigma^{l} \bar{\sigma}^{m} \sigma^{k} \bar{\theta}\right) \mathscr{D}_{l} v_{m k}+8[\bar{\theta} \bar{\lambda}, \theta \lambda] \\
& -4 i \theta \theta\left(\bar{\theta} \bar{\sigma}^{k} \sigma^{m} \partial_{k} \mathscr{D}_{m} \bar{\lambda}\right)-4 i \bar{\theta} \bar{\theta}\left(\theta \sigma^{k} \bar{\sigma}^{m} \partial_{k} \mathscr{D}_{m} \lambda\right) \\
& +8 i \bar{\theta} \bar{\theta}\left(\theta \sigma^{l} \bar{\sigma}^{m} \mathscr{D}_{l} \mathscr{D}_{m} \lambda\right)+8 i \bar{\theta} \bar{\theta}[\theta \lambda, d] \\
& +\theta \theta \bar{\theta} \bar{\theta}\left(2 \square d+2 i \partial^{m}\left[v_{m}, d\right]-i \operatorname{Tr}\left[\bar{\sigma}^{m} \sigma^{k} \bar{\sigma}^{n} \sigma^{l}\right] \partial_{n} \mathscr{D}_{l} v_{m k}\right. \\
& \left.+2 i \partial_{m}\left\{\left(\bar{\lambda} \bar{\sigma}^{m}\right)^{\alpha}, \lambda_{\alpha}\right\}\right) .
\end{aligned}
$$

Finally, we get the gauge covariant term in $(x, \theta, \bar{\theta})$ coordinates as follows.

$$
\begin{aligned}
\left(\frac{L_{\mathcal{V}}}{1-e^{-L_{\mathcal{V}}}} \cdot\left(D^{\alpha} w_{\alpha}+\left\{e^{-\mathcal{V}} D^{\alpha} e^{\mathcal{V}}, w_{\alpha}\right\}\right)\right)+\text { h.c. } \\
=-16 d+16 \theta \sigma^{m} \mathscr{D}_{m} \bar{\lambda}-16 \bar{\theta} \bar{\sigma}^{m} \mathscr{D}_{m} \lambda \\
\quad+16 \theta \sigma^{m} \bar{\theta} \mathscr{D}^{k} v_{m k}+16[\bar{\theta} \bar{\lambda}, \theta \lambda] \\
\quad-8 i \theta \theta\left(\bar{\theta} \bar{\sigma}^{l} \sigma^{m} \mathscr{D}_{l} \mathscr{D}_{m} \bar{\lambda}\right)+8 i \theta \theta[\bar{\theta} \bar{\lambda}, d] \\
\quad+8 i \bar{\theta} \bar{\theta}\left(\theta \sigma^{l} \bar{\sigma}^{m} \mathscr{D}_{l} \mathscr{D}_{m} \lambda\right)+8 i \bar{\theta} \bar{\theta}[\theta \lambda, d] \\
\quad+\theta \theta \bar{\theta} \bar{\theta}\left(4 \square d+4 i \partial^{m}\left[v_{m}, d\right]\right. \\
\quad+i \operatorname{Tr}\left[\bar{\sigma}^{m} \sigma^{l} \bar{\sigma}^{n} \sigma^{k}-\bar{\sigma}^{m} \sigma^{k} \bar{\sigma}^{n} \sigma^{l}\right] \mathscr{D}_{n} \mathscr{D}_{l} v_{m k} \\
\quad-2 i \partial_{m}\left\{\bar{\lambda}_{\dot{\alpha}},\left(\bar{\sigma}^{m} \lambda\right)^{\dot{\alpha}}\right\}+2 i \partial_{m}\left\{\left(\bar{\lambda} \bar{\sigma}^{m}\right)^{\alpha}, \lambda_{\alpha}\right\} \\
\left.\quad-\frac{4}{3}\left[v_{m},\left[v^{m}, d\right]\right]\right) .
\end{aligned}
$$


In a similar way, we obtain $\delta \mathcal{V}$ in terms of $(x, \theta, \bar{\theta})$ coordinates as

$$
\begin{aligned}
\delta \mathcal{V}(x, \theta, \bar{\theta})= & \Phi_{\mathcal{V}}+\Phi_{\mathcal{V}}^{\dagger}+\frac{1}{2}\left[\mathcal{V}, \Phi_{\mathcal{V}}-\Phi_{\mathcal{V}}^{\dagger}\right]+\frac{1}{12}\left[\mathcal{V},\left[\mathcal{V}, \Phi_{\mathcal{V}}+\Phi_{\mathcal{V}}^{\dagger}\right]\right] \\
= & 16 d-16 \theta \sigma^{m} \mathscr{D}_{m} \bar{\lambda}+16 \bar{\theta} \bar{\sigma}^{m} \mathscr{D}_{m} \lambda-16 \theta \sigma^{k} \bar{\theta} \mathscr{D}_{k} \partial_{m} v^{m} \\
& -8 i \theta \theta \bar{\theta} \bar{\sigma}^{k} \sigma^{m} \mathscr{D}_{k} \mathscr{D}_{m} \bar{\lambda}-8 \theta \theta \bar{\theta}_{\dot{\alpha}}\left[\bar{\lambda}^{\dot{\alpha}}, \partial_{m} v^{m}\right] \\
& -8 i \bar{\theta} \bar{\theta} \theta \sigma^{k} \bar{\sigma}^{m} \mathscr{D}_{k} \mathscr{D}_{m} \lambda-8 \bar{\theta} \bar{\theta} \theta^{\alpha}\left[\lambda_{\alpha}, \partial_{m} v^{m}\right] \\
& +4 \theta \theta \bar{\theta} \bar{\theta}\left(\square d+i\left\{\bar{\lambda}_{\dot{\alpha}},\left(\bar{\sigma}^{m} \mathscr{D}_{m} \lambda\right)^{\dot{\alpha}}\right\}-i\left\{\lambda^{\alpha},\left(\sigma^{m} \mathscr{D}_{m} \bar{\lambda}\right)_{\alpha}\right\}\right. \\
& \left.+i\left[d, \partial_{m} v^{m}\right]+i\left[v^{m}, \partial_{m} d\right]-\frac{1}{6}\left[v_{m},\left[v^{m}, d\right]\right]\right) .
\end{aligned}
$$

\subsection{Gradient flow equation of super Yang-Mills theory for each component of vector multiplet}

Because a physical quantity does not depend on the form of the $\alpha_{0}$ term, we choose a particular value $\alpha_{0}=1$. Then we obtain

$$
\begin{aligned}
\left(\frac{L_{\mathcal{V}}}{1-e^{-L_{\mathcal{V}}}} \cdot\left(D^{\alpha} w_{\alpha}+\left\{e^{-\mathcal{V}} D^{\alpha} e^{\mathcal{V}}, w_{\alpha}\right\}\right)\right)+h . c .+1 \cdot \delta \mathcal{V} \\
=16 \theta \sigma^{m} \bar{\theta} \mathscr{D}^{k} v_{m k}+16[\bar{\theta} \bar{\lambda}, \theta \lambda]-16 \theta \sigma^{k} \bar{\theta} \mathscr{D}_{k} \partial_{m} v^{m} \\
\quad-16 i \theta \theta \bar{\theta} \bar{\sigma}^{k} \sigma^{m} \mathscr{D}_{k} \mathscr{D}_{m} \bar{\lambda}+8 i \theta \theta\left[\bar{\theta} \bar{\lambda}, d+i \partial_{m} v^{m}\right] \\
\quad+16 i \bar{\theta} \bar{\theta} \theta \sigma^{k} \bar{\sigma}^{m} \mathscr{D}_{k} \mathscr{D}_{m} \lambda+8 i \bar{\theta} \bar{\theta}\left[\theta \lambda, d-i \partial_{m} v^{m}\right] \\
\quad+\theta \theta \bar{\theta} \bar{\theta}\left(8 \square d+8 i\left[v_{m}, \partial^{m} d\right]+i \operatorname{Tr}\left[\bar{\sigma}^{m} \sigma^{l} \bar{\sigma}^{n} \sigma^{k}-\bar{\sigma}^{m} \sigma^{k} \bar{\sigma}^{n} \sigma^{l}\right] \mathscr{D}_{n} \mathscr{D}_{l} v_{m k}\right. \\
\left.\quad+4 i\left\{\bar{\lambda}_{\dot{\alpha}},\left(\bar{\sigma}^{m} \mathscr{D}_{m} \lambda\right)^{\dot{\alpha}}\right\}-4 i\left\{\lambda^{\alpha},\left(\sigma^{m} \mathscr{D}_{m} \bar{\lambda}\right)_{\alpha}\right\}-2\left[v_{m},\left[v^{m}, d\right]\right]\right) .
\end{aligned}
$$

Finally, we obtain the flow equations for the each component of the vector multiplet as

$$
\begin{aligned}
\dot{c}= & 0, \\
\dot{\chi}= & 0, \\
\dot{\bar{\chi}}= & 0, \\
\dot{m}= & 0, \\
\dot{m}^{*}= & 0, \\
\dot{v}_{m}= & -16 \mathscr{D}^{k} v_{m k}+16 \mathscr{D}_{m} \partial_{k} v^{k}-8\left\{\bar{\lambda}_{\dot{\alpha}},\left(\bar{\sigma}_{m} \lambda\right)^{\dot{\alpha}}\right\}, \\
\dot{\bar{\lambda}}= & -16 \bar{\sigma}^{k} \sigma^{m} \mathscr{D}_{k} \mathscr{D}_{m} \bar{\lambda}+8\left[\bar{\lambda}, d+i \partial_{m} v^{m}\right], \\
\dot{\lambda}= & -16 \sigma^{k} \bar{\sigma}^{m} \mathscr{D}_{k} \mathscr{D}_{m} \lambda-8\left[\lambda, d-i \partial_{m} v^{m}\right], \\
\dot{d}= & 16 \square d+16 i\left[v_{m}, \partial^{m} d\right] \\
& +2 i \operatorname{Tr}\left[\bar{\sigma}^{m} \sigma^{l} \bar{\sigma}^{n} \sigma^{k}-\bar{\sigma}^{m} \sigma^{k} \bar{\sigma}^{n} \sigma^{l}\right] \mathscr{D}_{n} \mathscr{D}_{l} v_{m k} \\
& +8 i\left\{\bar{\lambda}_{\dot{\alpha}},\left(\bar{\sigma}^{m} \mathscr{D}_{m} \lambda\right)^{\dot{\alpha}}\right\}-8 i\left\{\lambda^{\alpha},\left(\sigma^{m} \mathscr{D}_{m} \bar{\lambda}\right)_{\alpha}\right\} \\
& -4\left[v_{m},\left[v^{m}, d\right]\right] .
\end{aligned}
$$


We find that the flow equations for each component are consistent with WZ gauge. Here we choose initial conditions to satisfy the $\mathrm{WZ}$ gauge at $t=0$ as

$$
\begin{aligned}
\left.c\right|_{t=0} & =0, \\
\left.\chi\right|_{t=0} & =0, \\
\left.\bar{\chi}\right|_{t=0} & =0, \\
\left.m\right|_{t=0} & =0, \\
\left.m^{*}\right|_{t=0} & =0, \\
\left.v_{m}\right|_{t=0} & =V_{m}, \\
\left.\bar{\lambda}\right|_{t=0} & =\bar{\Lambda}, \\
\left.\lambda\right|_{t=0} & =\Lambda, \\
\left.d\right|_{t=0} & =D .
\end{aligned}
$$

Let us compare the flow equation for Yang-Mills theory proposed by Luscher with our results for super Yang-Mills theory in eqs. (6.14) and (6.15). In ref. [4], Luscher claims that the gradient flow equations of the quark field are given as

$$
\begin{aligned}
& \dot{\bar{\chi}}=\bar{\chi} \overleftarrow{\Delta}+\alpha_{0} \bar{\chi} \partial_{\nu} B_{\nu} \\
& \dot{\chi}=\Delta \chi-\alpha_{0} \partial_{\nu} B_{\nu} \chi
\end{aligned}
$$

On the other hand our results for the gradient flow equations of the gaugino field in eqs. (6.14) and (6.15) are given as

$$
\begin{aligned}
& \dot{\bar{\lambda}}=-16 \bar{\sigma}^{k} \sigma^{m} \mathscr{D}_{k} \mathscr{D}_{m} \bar{\lambda}+8\left[\bar{\lambda}, d+i \partial_{m} v^{m}\right], \\
& \dot{\lambda}=-16 \sigma^{k} \bar{\sigma}^{m} \mathscr{D}_{k} \mathscr{D}_{m} \lambda-8\left[\lambda, d-i \partial_{m} v^{m}\right] .
\end{aligned}
$$

If we regard $\Delta$ as $\not D^{2}$, eqs. (6.26) and (6.27) are almost similar to our results eqs. (6.28) and (6.29) respectively except for $[\bar{\lambda}, d]$ term and $[\lambda, d]$ term and the point that $\alpha_{0}$ terms are described in terms of commutation relations.

\section{Summary and discussion}

In this paper, we proposed the generalized gradient flow equation for field theories with nonlinearly realized symmetry. Introducing the invariant norm for the variation of the field $\phi^{a}(x)$ where $a=1, \cdots, M$ is the index for the internal degrees of freedom, one can naturally define a metric $g_{a b}(\phi(x))$ in the functional space. Using this metric, we proposed the generalized gradient flow equation as

$$
\dot{\phi}_{t}^{a}(x)=-g^{a b}\left(\phi_{t}(x)\right) \frac{\delta S\left(\phi_{t}\right)}{\phi_{t}^{b}(x)} .
$$

Applying the generalized equation to super Yang-Mills theory using the superfield formalism, we obtained a gradient flow equation which manifestly preserves both super symmetry and super gauge symmetry. By choosing an appropriate $\alpha_{0}$ term described in terms of $\Phi_{\mathcal{V}}$ 
in eq. (5.3), we obtained a gradient flow equation of the super Yang-Mills theory which is closed under the WZ gauge.

We found that the gradient flow of the super Yang-Mills theory is very similar to the one in Yang-Mills theory and QCD. It is known that the gradient flow equation of the Yang-Mills theory and QCD has a wide variety of successful applications. We expect that our method may also be useful for testing the validity of various proposals of supersymmetric lattice models as well as extracting the physics of the super Yang-Mills theory. It is important to examine whether gauge invariant physical quantities require additional renormalization or not, which is under way. It is also interesting to study the properties of the generalized gradient flow equation for the nonlinear sigma model, which is a subject for future studies.

\section{Acknowledgments}

We would like to thank Masanori Hanada for useful advice. We also thank Satoshi Yamaguchi, Koji Hashimoto and Akinori Tanaka for fruitful discussions and comments. This work was supported by Grant-in-Aid for JSPS Fellows Grant Number 25.1336 and Grantin-Aid for Scientific Research (c) Grant Number 26400248.

\section{A Notation}

We use the following notation. The definition of the covariant derivative and the gauge field strength are

$$
\begin{aligned}
\mathscr{D}_{m} \cdot & \equiv \partial_{m} \cdot+\frac{i}{2}\left[v_{m}, \cdot\right], \\
v_{m n} & \equiv \partial_{m} v_{n}-\partial_{n} v_{m}+\frac{i}{2}\left[v_{m}, v_{n}\right] .
\end{aligned}
$$

respectively. The differential operators $D$ and $\bar{D}$ are

$$
\begin{aligned}
D_{\alpha}(x) & =\frac{\partial}{\partial \theta^{\alpha}}+i\left(\sigma^{m} \bar{\theta}\right)_{\alpha} \partial_{m}, \\
\bar{D}_{\dot{\alpha}}(x) & =-\frac{\partial}{\partial \bar{\theta}^{\dot{\alpha}}}-i\left(\theta \bar{\sigma}^{m}\right)_{\dot{\alpha}} \partial_{m},
\end{aligned}
$$

respectively. We introduce $y$ and $y^{\dagger}$ as

$$
\begin{aligned}
y^{m} & =x^{m}+i \theta \sigma^{m} \bar{\theta}, \\
y^{\dagger m} & =x^{m}-i \theta \sigma^{m} \bar{\theta} .
\end{aligned}
$$

respectively. For the sake of ease, we give $D$ and $\bar{D}$ in terms of $(y, \theta, \bar{\theta})$ or $\left(y^{\dagger}, \theta, \bar{\theta}\right)$ coordinates as

$$
\begin{aligned}
D_{\alpha}(y, \theta, \bar{\theta}) & =\frac{\partial}{\partial \theta^{\alpha}}+2 i\left(\sigma^{m} \bar{\theta}\right)_{\alpha} \frac{\partial}{\partial y^{m}}, \\
\bar{D}_{\dot{\alpha}}(y, \theta, \bar{\theta}) & =-\frac{\partial}{\partial \bar{\theta}^{\dot{\alpha}}}, \\
D_{\alpha}\left(y^{\dagger}, \theta, \bar{\theta}\right) & =\frac{\partial}{\partial \theta^{\alpha}}, \\
\bar{D}_{\dot{\alpha}}\left(y^{\dagger}, \theta, \bar{\theta}\right) & =-\frac{\partial}{\partial \bar{\theta}^{\dot{\alpha}}}-2 i\left(\theta \bar{\sigma}^{m}\right)_{\dot{\alpha}} \frac{\partial}{\partial y^{\dagger m}} .
\end{aligned}
$$




\section{B $O(N)$ nonlinear sigma model}

The $O(N)$ nonlinear sigma model is described by the unit $O(N)$ vector field $\phi^{\alpha}(x)(\alpha=$ $1, \cdots, N)$ with the constraint $\sum_{\alpha=1}^{N}\left(\phi^{\alpha}\right)^{2}=1$. The action is given as

$$
S=\frac{1}{2 \lambda} \int d^{D} x \sum_{\alpha=1}^{N} \partial_{\mu} \phi^{\alpha} \partial_{\mu} \phi^{\alpha},
$$

where $\lambda$ is the coupling. The action is invariant under the global $O(N)$ rotation

$$
\phi^{\alpha}(x) \rightarrow \phi^{\alpha}(x)+\sum_{\beta=1}^{N} \omega^{\alpha \beta} \phi^{\beta}
$$

where $\omega^{\alpha \beta}$ is an infinitesimal antisymmetric tensor. The invariant norm is given as

$$
\|\delta \phi\|^{2}=\int d^{D} x \sum_{\alpha=1}^{N}\left(\delta \phi^{\alpha}(x)\right)^{2} .
$$

The functional space can be parameterized by $\phi^{a}(x)(a=1, \cdots, N-1)$ as independent fields. Solving the constraint, the $N$-th component is expressed as

$$
\phi^{N}(x)= \pm\left[1-\sum_{a=1}^{N-1}\left(\phi^{a}(x)\right)^{2}\right]^{1 / 2} .
$$

Substituting eq. (B.4) into eq. (B.3), we obtain

$$
\|\delta \phi\|^{2}=\int d^{D} x \sum_{a, b=1}^{N-1} g_{a b}(\phi(x)) \delta \phi^{a}(x) \delta \phi^{b}(x),
$$

where the metric in the functional space $g_{a b}(\phi(x))$ is given by

$$
g_{a b}(\phi(x))=\delta_{a b}+\frac{\phi^{a}(x) \phi^{b}(x)}{1-\sum_{c=1}^{N-1}\left(\phi^{c}(x)\right)^{2}} .
$$

In this parameterization of the functional space, the $O(N)$ symmetry is nonlinearly realized as

$$
\begin{aligned}
\phi^{a}(x) \rightarrow \phi^{\prime a}(x) & =\phi^{a}(x)+\delta \phi^{a}(x) \\
& =\phi^{a}(x)+\sum_{b=1}^{N-1} \omega^{a b} \phi^{b}(x) \pm \omega^{a N}\left[1-\sum_{b=1}^{N-1}\left(\phi^{b}(x)\right)^{2}\right]^{1 / 2},
\end{aligned}
$$

where $\omega^{a b}, \omega^{a N}$ are the infinitesimal parameters for the $O(N)$ rotation. One can easily find that if one considers the transformation in eq. (B.7) as the coordinate transformation of the functional space, it is the isometry. In other words,

$$
g^{a b}\left(\phi^{\prime}\right)=\frac{\partial \phi^{\prime a}}{\partial \phi^{c}} \frac{\partial \phi^{\prime b}}{\partial \phi^{d}} g^{c d}(\phi)
$$


holds. It is then obvious that the following generalized equation

$$
\dot{\phi}_{t}^{a}(x)=-g^{a b}\left(\phi_{t}(x)\right) \frac{\delta S\left(\phi_{t}\right)}{\delta \phi_{t}^{b}(x)}
$$

gives essentially identical time evolution for $\phi$ and $\phi^{\prime}$. Moreover, a straightforward calculation shows that the gradient flow equation based on our proposal eq. (B.9) gives

$$
\dot{\phi}_{t}^{a}(x)=\frac{1}{\lambda}\left[\square \phi_{t}^{a}(x)-\phi_{t}^{a}(x)\left(\sum_{b=1}^{N-1} \phi_{t}^{b}(x) \square \phi_{t}^{b}(x)+\phi_{t}^{N}(x) \square \phi_{t}^{N}(x)\right)\right],
$$

with $\phi_{t}^{N}(x) \equiv \pm\left[1-\sum_{c=1}^{N-1}\left(\phi_{t}^{c}(x)\right)^{2}\right]^{1 / 2}$. The time evolution for $\phi_{t}^{N}(x)$ can also be induced using eq. (B.10) which reads

$$
\dot{\phi}_{t}^{N}(x)=\frac{1}{\lambda}\left[\square \phi_{t}^{N}(x)-\phi_{t}^{N}(x)\left(\sum_{b=1}^{N-1} \phi_{t}^{b}(x) \square \phi_{t}^{b}(x)+\phi_{t}^{N}(x) \square \phi_{t}^{N}(x)\right)\right] .
$$

Eqs. (B.10), (B.11) can be combined to

$$
\dot{\phi}^{\alpha}(x)=\frac{1}{\lambda}\left[\square \phi^{\alpha}(x)-\phi^{\alpha}(x)\left(\sum_{\beta=1}^{N} \phi^{\beta}(x) \square \phi^{\beta}(x)\right)\right],
$$

which is manifestly $O(N)$ symmetric and also keeps the constraint $\sum_{\alpha=1}^{N}\left(\phi^{\alpha}(x)\right)^{2}=1$.

\section{Lattice gauge theory}

The $\mathrm{SU}(N)$ lattice gauge theory is described by the link variable $\mathrm{U}(\mu, x)$ which are $N \times N$ $\mathrm{SU}(N)$ matrices . The action is given as

$$
S=\beta \sum_{x} \sum_{\mu>\nu}\left[1-\frac{1}{N} \operatorname{Tr}\left(P(\mu, \nu, x)+P^{\dagger}(\mu, \nu, x)\right)\right] .
$$

Here $P(\mu, \nu, x)$ is the plaquette defined as

$$
P(\mu, \nu, x)=\mathrm{U}(\mu, x) \mathrm{U}(\nu, x+\hat{\mu}) \mathrm{U}^{\dagger}(\mu, x+\hat{\nu}) \mathrm{U}^{\dagger}(\nu, x) .
$$

This action is invariant under the gauge transformation

$$
\mathrm{U}(\mu, x) \rightarrow \Lambda(x) \mathrm{U}(\mu, x) \Lambda^{\dagger}(x+\hat{\mu}),
$$

where $\Lambda(x)$ are arbitrary $\mathrm{SU}(N)$ matrices on the lattice site $x$ and the invariant norm is given as

$$
\|\delta U\|^{2}=\sum_{x} \sum_{\mu} \operatorname{Tr}\left[\delta \mathrm{U}^{\dagger}(\mu, x) \delta \mathrm{U}(\mu, x)\right] .
$$

The link variable $\mathrm{U}(\mu, x)$ can be parameterized as

$$
\mathrm{U}(\mu, x)=\exp \left(i A_{\mu}(x)\right),
$$


where $A_{\mu}(x) \equiv \sum_{a=1}^{N^{2}-1} A_{\mu}^{a}(x) T^{a}$ is $\mathrm{SU}(N)$ gauge field and $T^{a}\left(a=1, \cdots N^{2}-1\right)$ are traceless Hermitian $N \times N$ matrices with the condition $\operatorname{Tr}\left(T^{a} T^{b}\right)=\frac{1}{2} \delta^{a b}$.

The metric from the invariant norm can be explicitly obtained using the following matrix identity. Let $V$ be a $N \times N$ matrix and consider an infinitesimal variation $\delta V$. Defining an linear operator $L_{V}$ which acts on arbitrary matrix $M$ as

$$
L_{V} \cdot M \equiv[V, M]
$$

then the following matrix identity holds for linear order in $\delta V$

$$
e^{-V}\left(e^{V+\delta V}-e^{V}\right)=\frac{1-e^{-L_{V}}}{L_{V}} \cdot \delta V .
$$

Using this matrix identity and setting $V=i A_{\mu}(x)$, the invariant norm can be rewritten as

$$
\|\delta U\|^{2}=\sum_{x, \mu} \operatorname{Tr}\left[\left(\frac{1-e^{-L_{V}}}{L_{V}} \cdot T^{a}\right)\left(\frac{1-e^{-L_{V}}}{L_{V}} \cdot T^{b}\right)\right] \delta A_{\mu}^{a}(x) \delta A_{\mu}^{b}(x) .
$$

Therefore, the metric $g_{a b}\left(A_{\mu}(x)\right)$ becomes

$$
g_{a b}\left(A_{\mu}(x)\right)=\operatorname{Tr}\left[\left(\frac{1-e^{-L_{V}}}{L_{V}} \cdot T^{a}\right)\left(\frac{1-e^{-L_{V}}}{L_{V}} \cdot T^{b}\right)\right] .
$$

A simple algebra shows that the metric $g^{a b}\left(A_{\mu}(x)\right)$ is

$$
g^{a b}\left(A_{\mu}(x)\right)=4 \operatorname{Tr}\left[\left(\frac{L_{V}}{1-e^{-L_{V}}} \cdot T^{a}\right)\left(\frac{L_{V}}{1-e^{-L_{V}}} \cdot T^{b}\right)\right] .
$$

The generalized gradient flow equation for the field $A_{t \mu}(x)$

$$
\dot{A}_{t \mu}^{a}(x)=-g^{a b}\left(A_{\mu}(x)\right) \frac{\delta S\left(A_{t}\right)}{\delta A_{t \mu}^{b}(x)}
$$

gives

$$
\begin{aligned}
\dot{A}_{t \mu}(x)= & -i \beta \frac{L_{V}}{1-e^{-L_{V}}} \\
& \cdot\left(X_{t}(\mu, x) \mathrm{U}_{t}(\mu, x)-\mathrm{U}_{t}^{\dagger}(\mu, x) X_{t}^{\dagger}(\mu, x)\right. \\
& \left.-\frac{1}{N} \operatorname{tr}\left[X_{t}(\mu, x) \mathrm{U}_{t}(\mu, x)-\mathrm{U}_{t}^{\dagger}(\mu, x) X_{t}^{\dagger}(\mu, x)\right]\right)
\end{aligned}
$$

where $V_{t}, \mathrm{U}_{t}(\mu, x), X_{t}(\mu, x)$ are defined as

$$
\begin{aligned}
V_{t}= & i A_{t \mu}(x) \\
\mathrm{U}_{t}(\mu, x)= & \exp \left(i A_{t \mu}(x)\right) \\
X_{t}(\mu, x)= & \sum_{\nu \neq \mu}\left[\mathrm{U}_{t}(\nu, x+\hat{\mu}) \mathrm{U}_{t}^{\dagger}(\mu, x+\hat{\nu}) \mathrm{U}_{t}^{\dagger}(\nu, x)\right. \\
& \left.-\mathrm{U}_{t}^{\dagger}(\nu, x+\hat{\mu}-\hat{\nu}) \mathrm{U}_{t}^{\dagger}(\mu, x-\hat{\mu}) \mathrm{U}(\nu, x-\hat{\nu})\right] .
\end{aligned}
$$


Using eq. (C.12), we obtain the generalized gradient flow equation for $\mathrm{U}_{t}(\mu, x)$ as

$$
\begin{aligned}
\dot{U}_{t}(\mu, x)= & \beta\left(\mathrm{U}_{t}(\mu, x) X_{t}(\mu, x)-X_{t}^{\dagger}(\mu, x) U_{t}^{\dagger}(\mu, x)\right. \\
& \left.-\frac{1}{N} \operatorname{tr}\left[X_{t}(\mu, x) \mathrm{U}_{t}(\mu, x)-\mathrm{U}_{t}^{\dagger}(\mu, x) X_{t}^{\dagger}(\mu, x)\right]\right) \mathrm{U}_{t}(\mu, x) .
\end{aligned}
$$

Noting that

$$
\mathrm{U}_{t}(\mu, x) X_{t}(\mu, x)=\sum_{\nu \neq \mu} P_{t}(\mu, \nu, x)+P_{t}(\mu,-\nu, x)
$$

where $P_{t}(\mu, \nu, x)$ is the plaquette constructed from $\mathrm{U}_{t}$, the final form for the generalized gradient flow equation for the link field in $\mathrm{SU}(N)$ lattice gauge theory becomes

$$
\begin{aligned}
\dot{U}_{t}(\mu, x)= & \beta \sum_{\nu \neq \mu}\left(P_{t}(\mu, \nu, x)+P_{t}(\mu,-\nu, x)-P_{t}^{\dagger}(\mu, \nu, x)-P_{t}^{\dagger}(\mu,-\nu, x)\right. \\
& \left.-\frac{1}{N} \operatorname{Tr}\left(P_{t}(\mu, \nu, x)+P_{t}(\mu,-\nu, x)-P_{t}^{\dagger}(\mu, \nu, x)-P_{t}^{\dagger}(\mu,-\nu, x)\right)\right) \mathrm{U}_{t}(\mu, x)
\end{aligned}
$$

which agrees with eq. (1.4) given in the paper [1].

\section{Short summary of supersymmetry}

We give the notation of the superfield formalism. We follow the convention by Wess and Bagger [22].

\section{D.1 Definition}

The chiral superfield is defined by

$$
\bar{D}_{\alpha} \Phi=0 .
$$

We described chiral multiplet $\Phi=\{A, \psi, F\}$ in terms of $(x, \theta, \bar{\theta})$ coordinates as

$$
\begin{aligned}
\Phi(x, \theta, \bar{\theta})= & A+i \theta \sigma^{m} \bar{\theta} \partial_{m} A+\frac{1}{4} \theta \theta \bar{\theta} \bar{\theta} \square A \\
& +\sqrt{2} \theta \psi-\frac{i}{\sqrt{2}} \theta \theta \partial_{m} \psi \sigma^{m} \bar{\theta}+\theta \theta F .
\end{aligned}
$$

The vector superfield is defined by

$$
V=V^{\dagger}
$$

We described vector multiplet $V=\left\{C, X, \bar{X}, M, M^{*}, V_{m}, \Lambda, \bar{\Lambda}, D\right\}$ in terms of $(x, \theta, \bar{\theta})$ coordinates as

$$
\begin{aligned}
V(x, \theta, \bar{\theta})= & C+i \theta X-i \bar{\theta} \bar{X}+\frac{i}{2} \theta \theta M-\frac{i}{2} \bar{\theta} \bar{\theta} M^{*} \\
& -\theta \sigma^{m} \bar{\theta} V_{m}+i \theta \theta \bar{\theta}\left[\bar{\Lambda}+\frac{i}{2} \bar{\sigma}^{m} \partial_{m} X\right] \\
& -i \bar{\theta} \bar{\theta} \theta\left[\Lambda+\frac{i}{2} \sigma^{m} \partial_{m} \bar{X}\right]+\frac{1}{2} \theta \theta \bar{\theta} \bar{\theta}\left[D+\frac{1}{2} \square C\right] .
\end{aligned}
$$




\section{D.2 Wess-Zumino gauge}

The infinitesimal super gauge transformation is defined by

$$
V^{\prime}=V+\Phi+\Phi^{\dagger}
$$

Under this transformation, the each component of the vector multiplet transforms as follows:

$$
\begin{aligned}
C^{\prime} & =C+A+A^{*} \\
X^{\prime} & =X-i \sqrt{2} \psi \\
M^{\prime} & =M-2 i F \\
V_{m}^{\prime} & =V_{m}-i \partial_{m}\left(A-A^{*}\right) \\
\Lambda^{\prime} & =\Lambda \\
D^{\prime} & =D .
\end{aligned}
$$

Using this gauge transformation, we fixed the $\mathrm{WZ}$ gauge, which is $C, X, M=0$. Under this gauge, $\mathrm{V}$ is described in terms $(x, \theta, \bar{\theta})$ coordinates as

$$
\begin{aligned}
V(x, \theta, \bar{\theta}) & =-\theta \sigma^{m} \bar{\theta} V_{m}+i \theta \theta \bar{\theta} \bar{\Lambda}-i \bar{\theta} \bar{\theta} \Lambda+\frac{1}{2} \theta \theta \bar{\theta} \bar{\theta} D, \\
V^{2}(x, \theta, \bar{\theta}) & =-\frac{1}{2} \theta \theta \bar{\theta} \bar{\theta} V_{m} V^{m}, \\
V^{3}(x, \theta, \bar{\theta}) & =0 .
\end{aligned}
$$

And $\mathrm{V}$ is also described in terms $(y, \theta, \bar{\theta})$ coordinate as

$$
\begin{aligned}
V(y, \theta, \bar{\theta}) & =-\theta \sigma^{m} \bar{\theta} V_{m}+i \theta \theta \bar{\theta} \bar{\Lambda}-i \bar{\theta} \bar{\theta} \theta \Lambda+\frac{1}{2} \theta \theta \bar{\theta} \bar{\theta}\left[D-i \partial_{m} V^{m}\right], \\
V^{2}(y, \theta, \bar{\theta}) & =-\frac{1}{2} \theta \theta \bar{\theta} \bar{\theta} V_{m} V^{m}, \\
V^{3}(y, \theta, \bar{\theta}) & =0 .
\end{aligned}
$$

\section{E Derivation of gradient flow equation for vector superfield $\mathcal{V}$}

The invariant norm for the variation of the vector superfield $V$ is given as

$$
\|\delta V\|^{2}=-\int d^{8} z \operatorname{Tr}\left[e^{-V} \delta e^{V} e^{-V} \delta e^{V}\right] .
$$

The superfield $V$ can be expanded as

$$
V=V^{a} T^{a}
$$

where $T^{a}\left(a=1, \cdots, N^{2}-1\right)$ are the basis of $N \times N$ traceless Hermitian matrices with the condition $\operatorname{Tr}\left(T^{a} T^{b}\right)=\frac{1}{2} \delta^{a b}$. Using the matrix identity for infinitesimal variation $\delta V$

$$
e^{-V}\left(e^{V+\delta V}-e^{V}\right)=\frac{1-e^{-L_{V}}}{L_{V}} \cdot \delta V
$$


one can rewrite the invariant norm as follows:

$$
\|\delta V\|^{2}=-\int d^{8} z \delta V^{a}(z) \delta V^{b}(z) \operatorname{Tr}\left[\left(\frac{1-e^{-L_{V}}}{L_{V}} \cdot T^{a}\right)\left(\frac{1-e^{-L_{V}}}{L_{V}} \cdot T^{b}\right)\right] .
$$

Thus the metric $g_{a b}(V)$ is defined as

$$
g_{a b}(V)=-\operatorname{Tr}\left[\left(\frac{1-e^{-L_{V}}}{L_{V}} \cdot T^{a}\right)\left(\frac{1-e^{-L_{V}}}{L_{V}} \cdot T^{b}\right)\right] .
$$

The metric $g^{a b}(V)$, which is the inverse of the above is then defined as

$$
g^{a b}(V)=-4 \operatorname{Tr}\left[\left(\frac{L_{V}}{1-e^{-L_{V}}} \cdot T^{a}\right)\left(\frac{L_{V}}{1-e^{-L_{V}}} \cdot T^{b}\right)\right] .
$$

To derive $g^{a b}(V)$, we have used the matrix identity

$$
\operatorname{Tr}\left(A T^{a}\right) \operatorname{Tr}\left(T^{a} B\right)=\frac{1}{2} \operatorname{Tr}(A B)
$$

for arbitrary traceless matrices $A, B$.

The super Yang-Mills action is given as

$$
\begin{aligned}
S_{\mathrm{SYM}} & =-\int d^{4} x \int d^{2} \theta \operatorname{Tr}\left[W^{\alpha} W_{\alpha}\right]+\text { h.c. } \\
& =\int d^{8} z \operatorname{Tr}\left[e^{-V}\left(D^{\alpha} e^{V}\right) W_{\alpha}\right]+\text { h.c. }
\end{aligned}
$$

When we make a variation over the $V^{b}$ field, we obtain

$$
\begin{aligned}
\frac{\delta S_{\mathrm{SYM}}}{\delta V^{b}(z)}=\int d^{8} w \operatorname{Tr}\left[\frac{\delta}{\delta V^{a}(z)}\left\{e^{-V}\left(D^{\alpha} e^{V}\right) W_{\alpha}\right\}(w)\right]+\text { h.c. } \\
=2 \int d^{8} w \operatorname{Tr}\left[\frac{\delta e^{V(w)}}{\delta V^{b}(z)}\left\{\left(D^{\alpha} W_{\alpha}\right) e^{-V}+W^{\alpha}\left(D_{\alpha} e^{-V}\right)\right\}(w)\right. \\
\left.\quad-\frac{\delta e^{-V(w)}}{\delta V^{b}(z)}\left(D^{\alpha} e^{V}\right) W_{\alpha}(w)\right]+ \text { h.c. } \\
=\operatorname{Tr}\left[T^{b} \frac{e^{L_{V}}-1}{L_{V}} \cdot\left(D^{\alpha} W_{\alpha}+\left\{e^{-V} D^{\alpha} e^{V}, W_{\alpha}\right\}\right)(z)\right]+\text { h.c. }
\end{aligned}
$$

Here we used the useful formulae as

$$
\begin{aligned}
\delta\left(e^{V}\right) & =e^{V}\left[\frac{1-e^{-L_{V}}}{L_{V}} \cdot \delta V\right] \\
& =\left[\frac{e^{L_{V}}-1}{L_{V}} \cdot \delta V\right] e^{V}, \\
\delta\left(e^{-V}\right) & =e^{-V}\left[\frac{1-e^{L_{V}}}{L_{V}} \cdot \delta V\right] \\
& =\left[\frac{e^{-L_{V}}-1}{L_{V}} \cdot \delta V\right] e^{-V} .
\end{aligned}
$$


Combining eqs. (E.6) and (E.12), and replacing the $V$ field with the $\mathcal{V}$ field, we obtain

$$
\begin{aligned}
g^{a b}(\mathcal{V}) \frac{\delta S_{\mathrm{SYM}}}{\delta \mathcal{V}^{b}(z)}= & -4 \operatorname{Tr}\left[\left(\frac{L_{\mathcal{V}}}{1-e^{-L_{\mathcal{V}}}} \cdot T^{a}\right)\left(\frac{L_{\mathcal{V}}}{1-e^{-L_{\mathcal{V}}}} \cdot T^{b}\right)\right] \\
& \times \operatorname{Tr}\left[T^{b} \frac{e^{L_{\mathcal{V}}}-1}{L_{\mathcal{V}}} \cdot\left(D^{\alpha} w_{\alpha}+\left\{e^{-\mathcal{V}} D^{\alpha} e^{\mathcal{V}}, w_{\alpha}\right\}\right)(z)\right]+\text { h.c. } \\
= & -2 \operatorname{Tr}\left[T^{a} \frac{L_{\mathcal{V}}}{1-e^{-L_{\mathcal{V}}}} \cdot\left(D^{\alpha} w_{\alpha}+\left\{e^{-\mathcal{V}} D^{\alpha} e^{\mathcal{V}}, w_{\alpha}\right\}\right)(z)\right]+\text { h.c. }
\end{aligned}
$$

Here, we used the identity in eq. (E.7).

The matrix form of the gradient flow equation is

$$
\dot{\mathcal{V}}=-T^{a} g^{a b} \frac{\delta S_{\mathrm{SYM}}}{\delta \mathcal{V}^{b}}+\alpha_{0} \delta \mathcal{V}
$$

Using the matrix identity

$$
T^{a} \operatorname{Tr}\left[T^{a} A\right]=\frac{1}{2} A,
$$

for arbitrary traceless matrix $A$ and substituting eq. (E.18), we finally obtain

$$
\dot{\mathcal{V}}=\frac{L_{\mathcal{V}}}{1-e^{-L_{\mathcal{V}}}} \cdot\left(D^{\alpha} w_{\alpha}+\left\{e^{-\mathcal{V}} D^{\alpha} e^{\mathcal{V}}, w_{\alpha}\right\}\right)+\text { h.c. }+\alpha_{0} \delta \mathcal{V}
$$

\section{F Pure abelian supersymmetric theory}

We consider a supersymmetric pure Abelian gauge theory to simplify the discussion. Because this theory does not have an interaction, the theory also does not have divergences in the first place, but it is useful to understand the basic structure as a toy model.

\section{F.1 Derivation of gradient flow equation of pure abelian supersymmetric theory}

From the discussion in section 4, we obtain the gradient flow Equation of the pure Abelian supersymmetric theory. The free vector field action which is invariant under the supersymmetric gauge transformation is

$$
\begin{aligned}
S & =-\frac{1}{4} \int d^{4} x\left(\left.W^{\alpha} W_{\alpha}\right|_{\theta \theta}+\left.\bar{W}_{\dot{\alpha}} \bar{W}^{\dot{\alpha}}\right|_{\bar{\theta} \bar{\theta}}\right) \\
& =-\frac{1}{4} \int d^{8} z\left(D^{\alpha} W_{\alpha}+\bar{D}_{\dot{\alpha}} \bar{W}^{\dot{\alpha}}\right) V
\end{aligned}
$$

where $V$ is vector multiplet, $V=\left\{C, X, \bar{X}, M, M^{*}, V_{m}, \Lambda, \bar{\Lambda}, D\right\} . W$ and $\bar{W}$ are defined by

$$
\begin{aligned}
& W_{\alpha}=-\bar{D} \bar{D} D_{\alpha} V, \\
& \bar{W}_{\dot{\alpha}}=-D D \bar{D}_{\dot{\alpha}} V .
\end{aligned}
$$

Making variation of the action $S$ over $V$, we obtain

$$
\frac{\delta S}{\delta V}=-D^{\alpha} W_{\alpha}
$$


We used here the relation equation,

$$
D^{\alpha} W_{\alpha}=\bar{D}_{\dot{\alpha}} \bar{W}^{\dot{\alpha}} .
$$

Then we obtained the extended gradient flow equation of the pure supersymmetric theory as

$$
\begin{aligned}
& \dot{\mathcal{V}}=D^{\alpha} w_{\alpha}+\alpha_{0}\left(D^{2} \bar{D}^{2}+\bar{D}^{2} D^{2}\right) \mathcal{V}, \\
& \left.\mathcal{V}\right|_{t=0}=V,\left.\quad w_{\alpha}\right|_{t=0}=W_{\alpha} .
\end{aligned}
$$

where $\mathcal{V}$ is vector multiplet depending on the flow time, $\mathcal{V}=\left\{c, \chi, \bar{\chi}, m, m^{*}, v_{m}, \lambda, \bar{\lambda}, d\right\}$. The $\alpha_{0}$ term, which is the second term of the r.h.s. of eq. (F.6), is introduced to suppress the new gauge degrees of freedom under the evolution in the flow time.

\section{F.2 Gradient flow equation of pure Yang-Mills theory for each component of vector multiplet}

Describing the extended gradient flow equation in the coordinate of superspace which are labeled $(x, \theta, \bar{\theta})$, we find out the each dependence of the component of vector multiplet on the flow time.

$$
\begin{aligned}
\mathcal{V}(x, \theta, \bar{\theta})= & c+i \theta \chi-i \bar{\theta} \bar{\chi}+\frac{i}{2} \theta \theta m-\frac{i}{2} \bar{\theta} \bar{\theta} m^{*} \\
& -\theta \sigma^{m} \bar{\theta} v_{m}+i \theta \theta \bar{\theta}\left[\bar{\lambda}+\frac{i}{2} \bar{\sigma}^{m} \partial_{m} \chi\right] \\
& -i \bar{\theta} \bar{\theta} \theta\left[\lambda+\frac{i}{2} \sigma^{m} \partial_{m} \bar{\chi}\right]+\frac{1}{2} \theta \theta \bar{\theta} \bar{\theta}\left[d+\frac{1}{2} \square c\right] .
\end{aligned}
$$

Using (F.8), we calculate each terms of the gradient flow equation, we obtain

$$
\begin{aligned}
D^{\alpha} w_{\alpha}= & -2 d+2 \theta \sigma^{m} \partial_{m} \bar{\lambda}-2 \bar{\theta} \bar{\sigma}^{m} \partial_{m} \lambda+2\left(\theta \sigma^{k} \bar{\theta}\right) \partial^{m} v_{k m} \\
& -i \bar{\theta} \bar{\theta} \theta \square \lambda+i \theta \theta \bar{\theta} \square \bar{\lambda}+\frac{1}{2} \theta \theta \bar{\theta} \bar{\theta} \square d, \\
\left(D^{2} \bar{D}^{2}+\bar{D}^{2} D^{2}\right) \mathcal{V}= & 16(d+\square c)-16 \theta\left(\sigma^{m} \partial_{m} \bar{\lambda}-i \square \chi\right)+16 \bar{\theta}\left(\bar{\sigma}^{m} \partial_{m} \lambda-i \square \bar{\chi}\right) \\
& +8 i \theta \theta \square m-8 i \bar{\theta} \bar{\theta} \square m^{*}-16\left(\theta \sigma^{m} \bar{\theta}\right) \partial_{m} \partial^{k} v_{k} \\
& +8 i \theta \theta \bar{\theta}\left(\square \bar{\lambda}+i \bar{\sigma}^{m} \partial_{m} \square \chi\right)-8 i \bar{\theta} \bar{\theta} \theta\left(\square \lambda+i \sigma^{m} \partial_{m} \square \bar{\chi}\right) \\
& +4 \theta \theta \bar{\theta} \bar{\theta}(\square d+\square \square c) .
\end{aligned}
$$

Substituting (F.9) and (F.10) into (F.6), finally, we obtain the flow equations for the each component of the vector multiplet as

$$
\begin{aligned}
\dot{c} & =16 \alpha_{0} \square c-2\left(1-8 \alpha_{0}\right) d, \\
\dot{\chi} & =16 \alpha_{0} \square \chi-2 i\left(1-8 \alpha_{0}\right) \sigma^{m} \partial_{m} \bar{\lambda}, \\
\dot{\bar{\chi}} & =16 \alpha_{0} \square \bar{\chi}-2 i\left(1-8 \alpha_{0}\right) \bar{\sigma}^{m} \partial_{m} \lambda, \\
\dot{m} & =16 \alpha_{0} \square m, \\
\dot{m}^{*} & =16 \alpha_{0} \square m^{*}, \\
\dot{v}_{m} & =2 \square v_{m}-2\left(1-8 \alpha_{0}\right) \partial_{m} \partial^{k} v_{k}, \\
\dot{\bar{\lambda}} & =2 \square \bar{\lambda}, \\
\dot{\lambda} & =2 \square \lambda, \\
\dot{d} & =2 \square d .
\end{aligned}
$$


Taking $\alpha_{0}$ as

$$
\alpha_{0}=\frac{1}{8}
$$

we obtain

$$
\begin{aligned}
\dot{c} & =2 \square c, \\
\dot{\chi} & =2 \square \chi, \\
\dot{\bar{\chi}} & =2 \square \bar{\chi}, \\
\dot{m} & =2 \square m, \\
\dot{m}^{*} & =2 \square m^{*}, \\
\dot{v}_{m} & =2 \square v_{m}, \\
\dot{\bar{\lambda}} & =2 \square \bar{\lambda}, \\
\dot{\lambda} & =2 \square \lambda, \\
\dot{d} & =2 \square d .
\end{aligned}
$$

One can see that each component of the vector multiplet evolves separately in time.

\section{F.3 Flow time dependence of super gauge transformation}

When we demand that the gradient flow equation (F.6) is invariant under the super gauge transformation,

$$
\mathcal{V}^{\prime}=\mathcal{V}+\phi+\phi^{\dagger}
$$

at each time, $\phi$ have to satisfy the equation as

$$
\begin{aligned}
\dot{\phi} & =\alpha_{0} \bar{D}^{2} D^{2} \phi, \\
\left.\phi\right|_{t=0} & =\Phi,
\end{aligned}
$$

where $\Phi$ is a chiral field,

$$
\bar{D} \Phi=0 .
$$

The chirality of the $\phi$ at each flow time is guaranteed by eq. (F.31).

\section{G Expansion of equation (6.1) with component fields}

For the convenience of the expansion of (6.1) with the component fields, we give useful methods and formulae.

\section{G.1 Coordinate transformation}

It is useful to calculate $w_{\alpha}$ in terms of $(y, \theta, \bar{\theta})$ coordinates. We obtain $w_{\alpha}$ as

$$
\begin{aligned}
w_{\alpha}(y, \theta, \bar{\theta})= & -\bar{D}^{2}\left(e^{-\mathcal{V}} D_{\alpha} e^{\mathcal{V}}\right) \\
= & -4 i \lambda_{\alpha}+4 \theta_{\alpha} d-2 i\left(\sigma^{m} \bar{\sigma}^{k} \theta\right)_{\alpha} v_{m k} \\
& +4 \theta \theta\left\{\sigma^{m} \mathscr{D}_{m} \bar{\lambda}\right\}_{\alpha} .
\end{aligned}
$$

Using the expansion formula,

$$
f(y, \theta, \bar{\theta})=f(x)+i \theta \sigma^{m} \bar{\theta} \partial_{m} f(x)+\frac{1}{4} \theta \theta \bar{\theta} \bar{\theta} \square f(x),
$$


and

$$
f(x, \theta, \bar{\theta})=f(y)-i \theta \sigma^{m} \bar{\theta} \partial_{m} f(y)+\frac{1}{4} \theta \theta \bar{\theta} \bar{\theta} \square f(y),
$$

we always rewrite the results in the $(y, \theta, \bar{\theta})$ coordinate or $(x, \theta, \bar{\theta})$ either. For example,

$$
\begin{aligned}
w_{\alpha}(x, \theta, \bar{\theta})= & -4 i \lambda_{\alpha}+4 \theta_{\alpha} d-2 i\left(\sigma^{m} \bar{\sigma}^{k} \theta\right)_{\alpha} v_{m k} \\
& +4 \theta \theta\left\{\sigma^{m} \mathscr{D}_{m} \bar{\lambda}\right\}_{\alpha}+4\left(\theta \sigma^{m} \bar{\theta}\right) \partial_{m} \lambda_{\alpha} \\
& +2 \theta \theta\left(\sigma^{m} \bar{\theta}\right)_{\alpha}\left\{-i \partial_{m} d+\partial_{m} \partial^{k} v_{k}-\square v_{m}\right\} \\
& +\frac{i}{2} \theta \theta\left(\sigma^{m} \bar{\sigma}^{k} \sigma^{l} \bar{\theta}\right)_{\alpha} \partial_{l}\left[v_{k}, v_{m}\right]-i \theta \theta \bar{\theta} \bar{\theta} \square \lambda_{\alpha} .
\end{aligned}
$$

Note that they are not covariant under the super gauge transformation, because we take the WZ gauge fixing. Using (A.7), we obtain the result of calculation of $D^{\alpha} w_{\alpha}$ which is first term of the r.h.s. of (6.1) as

$$
\begin{aligned}
D^{\alpha} w_{\alpha}(y, \theta, \bar{\theta})= & -8 d+8 \theta \sigma^{m} \mathscr{D}_{m} \bar{\lambda}-8 \bar{\theta} \bar{\sigma}^{m} \partial_{m} \lambda \\
& -8 i\left(\bar{\theta} \bar{\sigma}^{m} \theta\right) \partial_{m} d-4\left(\bar{\theta} \bar{\sigma}^{l} \sigma^{m} \bar{\sigma}^{k} \theta\right) \partial_{l} v_{m k} \\
& -8 i \theta \theta\left\{\bar{\theta} \bar{\sigma}^{l} \sigma^{m} \partial_{l} \mathscr{D}_{m} \bar{\lambda}\right\} .
\end{aligned}
$$

\section{G.2 Useful formulae}

We also give useful formulae to obtain the second term with component field of r.h.s. of (6.1) in terms of $(y, \theta, \bar{\theta})$ coordinates as

$$
\begin{aligned}
e^{-\mathcal{V}} D^{\alpha} e^{\mathcal{V}}(y, \theta, \bar{\theta})= & \left(\bar{\theta} \bar{\sigma}^{m}\right)^{\alpha} v_{m}+2 i \theta^{\alpha} \bar{\theta} \bar{\lambda}-i \bar{\theta} \bar{\theta} \lambda^{\alpha} \\
& +\bar{\theta} \bar{\theta}\left(\theta^{\alpha} d-\frac{i}{2}\left(\theta \sigma^{m} \bar{\sigma}^{k}\right)^{\alpha} v_{k m}\right) \\
& -\theta \theta \bar{\theta} \bar{\theta} \mathscr{D}_{m}\left(\bar{\lambda} \bar{\sigma}^{m}\right)^{\alpha} .
\end{aligned}
$$

Finally we obtain the $A$ in terms of $(y, \theta, \bar{\theta})$ coordinates as

$$
\begin{aligned}
\left(D^{\alpha} w_{\alpha}+\left\{e^{-\mathcal{V}} D^{\alpha} e^{\mathcal{V}}, w_{\alpha}\right\}\right)(y, \theta, \bar{\theta})= & -8 d+8 \theta \sigma^{m} \mathscr{D}_{m} \bar{\lambda}-8 \bar{\theta} \bar{\sigma}^{m} \mathscr{D}_{m} \lambda \\
& +8[\bar{\theta} \bar{\lambda}, \theta \lambda]-8 i\left(\bar{\theta} \bar{\sigma}^{m} \theta\right) \mathscr{D}_{m} d \\
& +4\left(\theta \sigma^{k} \bar{\sigma}^{m} \sigma^{l} \bar{\theta}\right) \mathscr{D}_{l} v_{m k} \\
& -8 i \theta \theta\left(\bar{\theta} \bar{\sigma}^{l} \sigma^{m} \mathscr{D}_{l} \mathscr{D}_{m} \bar{\lambda}\right) \\
& +8 i \theta \theta[\bar{\theta} \bar{\lambda}, d] .
\end{aligned}
$$

The $A^{\dagger}$ in terms of $\left(y^{\dagger}, \theta, \bar{\theta}\right)$ coordinates is

$$
\begin{aligned}
\left(D^{\alpha} w_{\alpha}+\left\{e^{-\mathcal{V}} D^{\alpha} e^{\mathcal{V}}, w_{\alpha}\right\}\right)^{\dagger}\left(y^{\dagger}, \theta, \bar{\theta}\right)= & -8 d-8 \bar{\theta} \bar{\sigma}^{m} \mathscr{D}_{m} \lambda+8 \theta \sigma^{m} \mathscr{D}_{m} \bar{\lambda} \\
& +8[\bar{\theta} \bar{\lambda}, \theta \lambda]+8 i\left(\bar{\theta} \bar{\sigma}^{m} \theta\right) \mathscr{D}_{m} d \\
& +4\left(\theta \sigma^{l} \bar{\sigma}^{m} \sigma^{k} \bar{\theta}\right) \mathscr{D}_{l} v_{m k} \\
& +8 i \bar{\theta} \bar{\theta}\left(\mathscr{D}_{l} \mathscr{D}_{m} \lambda \sigma^{m} \bar{\sigma}^{l} \theta\right) \\
& +8 i \bar{\theta} \bar{\theta}\left[\lambda\left(y^{\dagger}\right) \theta, d\right] .
\end{aligned}
$$


Open Access. This article is distributed under the terms of the Creative Commons Attribution License (CC-BY 4.0), which permits any use, distribution and reproduction in any medium, provided the original author(s) and source are credited.

\section{References}

[1] M. Lüscher, Properties and uses of the Wilson flow in lattice QCD, JHEP 08 (2010) 071 [arXiv: 1006.4518] [INSPIRE].

[2] M. Lüscher and P. Weisz, Perturbative analysis of the gradient flow in non-abelian gauge theories, JHEP 02 (2011) 051 [arXiv:1101.0963] [INSPIRE].

[3] M. Lüscher, Future applications of the Yang-Mills gradient flow in lattice QCD, arXiv: 1308.5598 [INSPIRE].

[4] M. Lüscher, Chiral symmetry and the Yang-Mills gradient flow, JHEP 04 (2013) 123 [arXiv: 1302.5246] [INSPIRE].

[5] H. Suzuki, Energy-momentum tensor from the Yang-Mills gradient flow, Prog. Theor. Exp. Phys. 2013 (2013) 083B03 [arXiv: 1304.0533] [InSPIRE].

[6] L. Del Debbio, A. Patella and A. Rago, Space-time symmetries and the Yang-Mills gradient flow, JHEP 11 (2013) 212 [arXiv:1306.1173] [InSPIRE].

[7] Z. Fodor, K. Holland, J. Kuti, D. Nogradi and C.H. Wong, The Yang-Mills gradient flow in finite volume, JHEP 11 (2012) 007 [arXiv:1208.1051] [INSPIRE].

[8] Z. Fodor, K. Holland, J. Kuti, D. Nogradi and C.H. Wong, The gradient flow running coupling scheme, PoS(LATTICE 2012) 050 [arXiv:1211.3247] [INSPIRE].

[9] P. Fritzsch and A. Ramos, The gradient flow coupling in the Schrödinger Functional, JHEP 10 (2013) 008 [arXiv: 1301.4388] [INSPIRE].

[10] P. Fritzsch and A. Ramos, Studying the gradient flow coupling in the Schrödinger functional, PoS(Lattice 2013) 319 [arXiv: 1308.4559] [INSPIRE].

[11] A. Ramos, The gradient flow in a twisted box, PoS (Lattice 2013) 053 [arXiv:1308.4558] [INSPIRE].

[12] FlowQCD collaboration, M. Asakawa, T. Hatsuda, E. Itou, M. Kitazawa and H. Suzuki, Thermodynamics of SU(3) Gauge Theory from Gradient Flow,

Phys. Rev. D 90 (2014) 011501(R) [arXiv:1312.7492] [INSPIRE].

[13] M. Lüscher, Step scaling and the Yang-Mills gradient flow, JHEP 06 (2014) 105 [arXiv: 1404.5930] [INSPIRE].

[14] J. Rantaharju, The Gradient Flow Coupling in Minimal Walking Technicolor, PoS(Lattice 2013) 084 [arXiv: 1311.3719] [INSPIRE].

[15] H. Makino and H. Suzuki, Lattice energy-momentum tensor from the Yang-Mills gradient flow - a simpler prescription, arXiv:1404.2758 [INSPIRE].

[16] H. Makino and H. Suzuki, Lattice energy-momentum tensor from the Yang-Mills gradient flow-inclusion of fermion fields, Prog. Theor. Exp. Phys. 2014 (2014) $063 \mathrm{~B} 02$ [arXiv: 1403.4772] [INSPIRE]. 
[17] P. Fritzsch, A. Ramos and F. Stollenwerk, Critical slowing down and the gradient flow coupling in the Schrödinger functional, PoS (Lattice 2013) 461 [arXiv:1311.7304] [INSPIRE].

[18] O. Bär and M. Golterman, Chiral perturbation theory for gradient flow observables, Phys. Rev. D 89 (2014) 034505 [arXiv: 1312.4999] [INSPIRE].

[19] M. Dalla Brida and D. Hesse, Numerical Stochastic Perturbation Theory and the Gradient Flow, PoS (Lattice 2013) 326 [arXiv:1311.3936] [INSPIRE].

[20] C. Monahan and K. Orginos, Finite volume renormalization scheme for fermionic operators, PoS (Lattice 2013) 443 [arXiv: 1311.2310] [INSPIRE].

[21] A. Shindler, Chiral Ward identities, automatic $O(a)$ improvement and the gradient flow, Nucl. Phys. B 881 (2014) 71 [arXiv:1312.4908] [INSPIRE].

[22] J. Wess and J. Bagger, Supersymmetry and Supergravity, Princeton Serieis in Physics, second edition revised and expanded, Princeton University Press, Princeton New Jersey (1992).

[23] G. Robert, Covariant Formulation of Non-Equilibrium Statistical Thermodynamics, Z. Physik B 26 (1977) 397.

[24] G. Robert, Covariant stochastic calculus in the sense of Itô, Phys. Lett. A 109 (1985) 209.

[25] M. Namiki, I. Ohba and K. Okano, Stochastic Quantization of Constrained Systems: General Theory and Nonlinear $\sigma$ Model, Prog. Theor. Phys. 72 (1984) 350 [InSPIRE].

[26] H. Rumpf, Stochastic Quantization of Einstein Gravity, Phys. Rev. D 33 (1986) 942 [INSPIRE].

[27] J. Zinn-Justin, Renormalization and Stochastic Quantization, Nucl. Phys. B 275 (1986) 135 [INSPIRE].

[28] M.B. Halpern, Coordinate-invariant regularization, Annals Phys. 178 (1987) 272 [InSPIRE].

[29] N. Nakazawa and D. Ennyu, Background Field Method for Nonlinear $\sigma$ Model in Stochastic Quantization, Nucl. Phys. B 305 (1988) 516 [INSPIRE].

[30] N. Nakazawa, The Extended Local Gauge Invariance and the BRS Symmetry in Stochastic Quantization of Gauge Fields, Nucl. Phys. B 335 (1990) 546 [INSPIRE].

[31] N. Nakazawa, $N=1$ supersymmetric Yang-Mills theory in Itō calculus, Prog. Theor. Phys. 110 (2004) 1117 [INSPIRE].

[32] N. Nakazawa, Stochastic gauge fixing in $N=1$ supersymmetric Yang-Mills theory, Prog. Theor. Phys. 116 (2007) 883 [hep-th/0308081] [INSPIRE]. 